\title{
Morphology and strain-induced defect structure of ultrathin epitaxial Fe films on Mo(110)
}

\author{
S. Murphy, ${ }^{*}$ D. Mac Mathúna, G. Mariotto, and I. V. Shvets \\ SFI Laboratory, Department of Physics, Trinity College, University of Dublin, Dublin 2, Ireland \\ (Received 10 June 2002; revised manuscript received 23 August 2002; published 27 November 2002)
}

\begin{abstract}
Fe films in a coverage range of $0.4 \leqslant \theta \leqslant 4.7 \mathrm{ML}$ were deposited on a Mo(110) substrate in the $300 \leqslant T$ $\leqslant 700 \mathrm{~K}$ temperature range. It is found that growth around $300 \mathrm{~K}$ is mediated by the step-flow growth mechanism, in contrast with previous studies of the $\mathrm{Fe} / \mathrm{Mo}(110)$ and $\mathrm{Fe} / \mathrm{W}(110)$ systems, where growth at 300 $\mathrm{K}$ was mediated by two-dimensional island nucleation and coalescence. This difference is attributed to the slightly higher substrate temperature (between 300 and $345 \mathrm{~K}$ ) during deposition. A transition from layer-bylayer to Stranski-Krastanov growth is observed in films grown in the $300 \leqslant T \leqslant 345 \mathrm{~K}$ range at around a $1.8 \mathrm{ML}$ coverage. Strain-relieving dislocation defects appear along the [001] direction in the second Fe layer and develop with increasing film thickness into a dislocation network at around a 2.4 ML coverage. The dislocation defects in the second Fe layer act as preferential nucleation sites for third layer islands. At elevated temperatures $(495 \leqslant T \leqslant 700 \mathrm{~K})$, the first and second Fe layers are formed by the step-flow growth mechanism. Subsequent coverages are characterized by the formation of distinctive wedge-shaped islands supported on an Fe monolayer. A two-dimensional dislocation network is formed in the fourth Fe layer of these islands, from an array of closely-spaced dislocation lines in the third layer. Similar to the Fe/W(110) system, the magnetic properties of these films are expected to vary significantly on the nanometer scale and they are therefore potential candidates for spin-polarized scanning tunneling microscopy studies.
\end{abstract}

DOI: 10.1103/PhysRevB.66.195417

PACS number(s): 68.55.Ac, 68.55.Jk

\section{INTRODUCTION}

Ultrathin epitaxial film systems exhibit a variety of interesting properties due to the strong correlation between the electronic structure of the film and its morphology, strain, and defect structure. ${ }^{1-3}$ For example, the magnetic properties of heteroepitaxial ultrathin $\mathrm{Fe}$ and $\mathrm{Ni}$ films are sensitive to the mechanical strain imposed on the film by lattice mismatch with the underlying substrate. The thicknessdependent behavior of the magnetic properties in the $\mathrm{Ni}$ / $\mathrm{Cu}(001)$ system is a good example. ${ }^{4-6}$

A sharp transition from an in-plane to an out-of-plane magnetic easy axis is induced in clean pseudomorphic $\mathrm{Ni}$ films at a coverage $\theta$ of around $10-11 \mathrm{ML}$ (monolayers). ${ }^{6} \mathrm{~A}$ tetragonal distortion is produced in the initial layers of the film as it is forced into registry with the substrate. This produces a magnetoelastic anisotropy that overcomes the combined shape, surface and interface anisotropies present in the film, to force a perpendicular magnetic easy axis. As the film grows thicker, the magneto-elastic contribution is reduced as strain-relieving dislocations are formed in the film. This leads to a second, gradual reorientation of the easy axis back into the film plane over coverages of 40-50 ML. A strain-induced transition is also found in the Fe/W(110) system. $^{7}$ However, with a significantly larger lattice mismatch than is present in $\mathrm{Ni} / \mathrm{Cu}(001)$ growth $\left[\left(a_{W}\right.\right.$ $\left.-a_{F e}\right) / a_{F e}=10.4 \%$ homogeneous tensile strain for $\mathrm{Fe} /$ $\mathrm{W}(110)$ compared to $\left(a_{C u}-a_{N i}\right) / a_{N i}=2.6 \%$ for $\mathrm{Ni} /$ $\mathrm{Cu}(001)]$, this transition is found within only the first few monolayers of $\mathrm{Fe} / \mathrm{W}(110)$ growth.

Fe films grown on $\mathrm{W}(110)$ at $300 \mathrm{~K}$ begin as an array of unconnected, monolayer-thick islands, which are superparamagnetic at $115 \mathrm{~K} .{ }^{8}$ At coverages greater than 0.6 ML, ferromagnetic order is induced by magnetic percolation through island coalescence. The closed first $\mathrm{Fe}$ layer becomes ferromagnetic below $225 \mathrm{~K},{ }^{8}$ with an in-plane easy axis of magnetization along the [1 $1 \overline{0} 0$ direction. ${ }^{8,9}$ Defectfree second layer islands are formed on top of this layer in the $1.0 \leqslant \theta \leqslant 1.5 \mathrm{ML}$ coverage range, which have a straininduced out-of-plane magnetization and are ferromagnetic at $300 \mathrm{~K}^{7}$ As the film coverage increases above $1.5 \mathrm{ML}$, the average $\mathrm{Fe}$ island area also increases and dislocations begin to form. The resulting strain relief causes a reorientation of the magnetic easy axis back into the film plane.

This behavior is also observed in Fe films grown on $\mathrm{W}(110)$ at elevated temperatures $(570 \leqslant T \leqslant 700 \mathrm{~K})$. In the $1.2 \leqslant \theta \leqslant 1.5 \mathrm{ML}$ coverage range, Fe films form as arrays of alternating monolayer/double-layer stripes by the step-flow growth mechanism. ${ }^{10}$ The double-layer thick stripes have an out-of-plane magnetization and are antiferromagnetically coupled to one another by dipolar interactions. ${ }^{10}$ This arrangement was recently resolved using spin-polarized scanning tunneling spectroscopy. ${ }^{11,12}$ Gas adsorption onto the film critically affects this magnetic behavior, as even slight exposure to residual gases under ultra-high vacuum conditions can force a reorientation of the strain-induced magnetic easy axis back into the film plane. ${ }^{13,14}$ In addition, the film morphology and consequent magnetic behavior are affected by the underlying substrate step orientation. For submonolayer growth of $\mathrm{Fe}$ on W(110) at $700 \mathrm{~K}$, the film switches between step-flow growth along [001] oriented steps to the formation of chains of two-dimensional triangular islands along [1 $\overline{1} 0]$ oriented steps. ${ }^{15}$ Because the $\mathrm{Fe} / \mathrm{W}(110)$ system exhibits such a variety of interesting phenomena, it is instructive to investigate the $\mathrm{Fe} / \mathrm{Mo}(110)$ system, which is similar to the $\mathrm{Fe} / \mathrm{W}(110)$ system in terms of lattice mismatch $\left(a_{M o}-a_{F e} / a_{F e}=9.8 \%\right)$ and surface free energies $\quad\left[\gamma_{\mathrm{Fe}}=2.55 \mathrm{~J} \mathrm{~m}^{-2}, \gamma_{W}=3.3 \mathrm{~J} \mathrm{~m}^{-2}\right.$, and $\gamma_{M o}$ $=2.95 \mathrm{~J} \mathrm{~m}^{-2}$ ] (Ref. 16). The Fe/Mo(110) system has so far been studied to a far lesser extent than $\mathrm{Fe} / \mathrm{W}(110),{ }^{17-19}$ so 
that less information is available on the magnetic and structural properties of this system. This structural information is readily accessible through scanning tunneling microscopy (STM) and low-energy electron diffraction (LEED). The aim of this study is to investigate the growth modes of the $\mathrm{Fe} / \mathrm{Mo}(110)$ system and, in particular, to establish the role of strain caused by the film-substrate lattice mismatch. We compare these results with previous results obtained on the $\mathrm{Fe} / \mathrm{Mo}(110)$ and $\mathrm{Fe} / \mathrm{W}(110)$ epitaxial systems. Finally, we will discuss the possibility of using $\mathrm{Fe} / \mathrm{Mo}(110)$ as a test sample for spin-polarized STM experiments.

\section{EXPERIMENT}

Sample preparation and analysis were performed in an ultrahigh vacuum (UHV) system with a base pressure in the mid $10^{-11}$ mbar. A cylindrical mirror analyzer was used for Auger electron spectroscopy (AES), while LEED was performed with a four-grid retarding-field analyzer. STM was performed using a home-built room-temperature instrument, incorporating a piezotube scanner for tip positioning and a piezowalker approach mechanism similar to that discussed by Mariotto and co-workers. ${ }^{20,21}$ All STM images were obtained using $\mathrm{W}$ tips and with a typical tunnel current of $I_{T}=0.1 \mathrm{nA}$ and sample bias of $V_{T}=30 \mathrm{mV}$. The Mo(110) substrate was prepared from a $4 N$ purity single crystal, with a misorientation of $0.65^{\circ}$ off the (110) plane, yielding an average terrace width of the order of $200 \AA$. The step direction was found to run parallel to the [11ㅣ crystallographic direction. The substrate was cleaned by alternate cycles of oxidation and flash annealing in an electron-beam heater. In each cycle, the surface was first annealed at 1300 $\leqslant \mathrm{T} \leqslant 1550 \mathrm{~K}$ (measured by infrad pyrometer) in a 7 $\times 10^{-7}$ mbar oxygen atmosphere for 30-60 min cycles. The resulting oxide layer was then removed from the surface by repeated flash annealing to $2400 \mathrm{~K}$ for $10-15 \mathrm{sec}$ in UHV. This procedure was repeated until the carbon and oxygen impurity levels were below the detection limit of the AES setup $(<1$ at.\%) and the surface produced a sharp $1 \times 1$ LEED pattern consistent with the bulk termination of the Mo(110) surface. Typically, the sample was cleaned 30-40 min prior to each deposition. The sample stage was equipped with a resistive heater and thermocouple so that deposition could be carried out at elevated substrate temperatures. The accuracy of the temperature control of the sample during the deposition was generally better than $\pm 15 \mathrm{~K}$. Fe films were deposited by electron beam evaporation of a $99.9 \%$ purity $\mathrm{Fe}$ rod, which was outgassed prior to each deposition. The deposition flux was monitored during deposition using a quartz crystal balance. The chamber pressure during evaporation did not rise above $3.5 \times 10^{-10} \mathrm{mbar}$, while evaporation rates were typically $0.006 \leqslant D \leqslant 0.03 \AA . s^{-1}$. Film coverages $\theta$ are described in pseudomorphic monolayers, where $\theta=1$ for $1.43 \times 10^{19} \mathrm{Fe}$ atoms $\mathrm{m}^{-2}$ corresponds to the atomic packing density of a $\mathrm{Mo}(110)$ plane. Where an accurate measurement was possible, film coverages were determined directly from STM images; otherwise coverages were determined from the deposition flux measured by the quartz crystal balance.

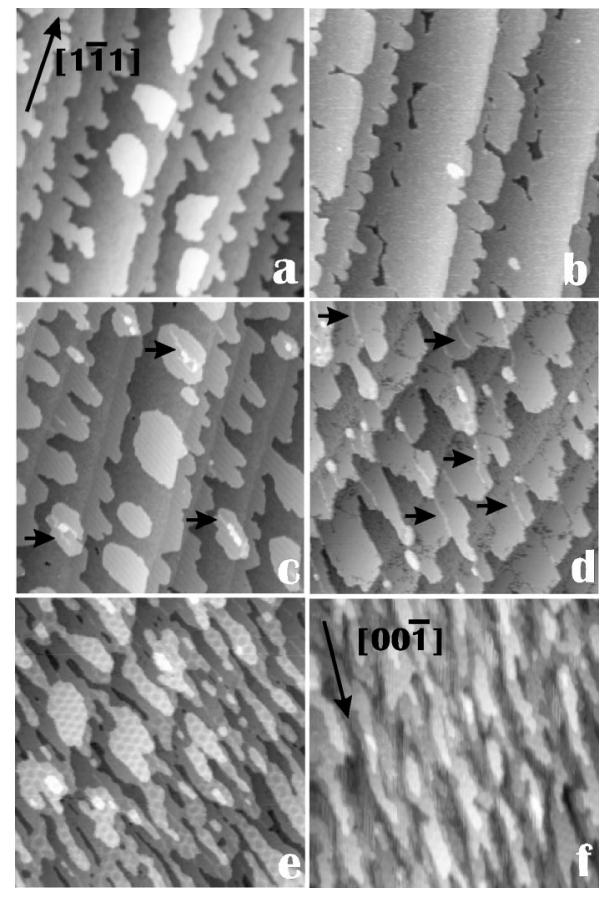

FIG. 1. $1000 \times 1000 \AA^{2}$ STM images of Fe films grown on $\mathrm{Mo}(110)$ near room temperature. (a) $0.4 \mathrm{ML}$ at $330 \pm 15 \mathrm{~K}$, (b) 0.9 $\mathrm{ML}$ at $300 \pm 15 \mathrm{~K}$, (c) $1.6 \mathrm{ML}$ at $330 \pm 15 \mathrm{~K}$, (d) $1.8 \mathrm{ML}$ at 340 $\pm 15 \mathrm{~K}$, (e) $2.4 \mathrm{ML}$ at $325 \pm 15 \mathrm{~K}$, and (f) $4.7 \mathrm{ML}$ at $320 \pm 15 \mathrm{~K}$. In each image, the [111] (step-edge) direction runs from the bottomleft to upper-right corner of the image. The arrows in (c) and (d) mark the position of dislocation lines propagating along the [001] direction in the second Fe layer.

\section{RESULTS AND DISCUSSION}

\section{A. Room-temperature growth}

The sequence of growth near room temperature, with increasing film coverage, is displayed in Fig. 1. The first and second layers grow in a layer-by-layer fashion, while higher coverages are deposited in a layer-plus-island (StranskiKrastanov) fashion. At higher coverages, there is increasingly preferential growth along the [001] direction with an elongation of islands along this direction. This phenomenon was encountered in other bcc (110) epitaxial systems, ${ }^{22-24}$ and was attributed to both anisotropic sticking probabilities $^{22}$ and the local diffusion behavior at the island edges. ${ }^{24}$ Other factors which may contribute to the island shape are anisotropic surface diffusion due to the two fold symmetry of the bcc (110) surface $e^{25}$ and stress anisotropy within the film, due to the higher cost in elastic energy required to pseudomorphically strain an Fe layer along the [1 $\overline{1} 0]$ direction compared to the $[00 \overline{1}]$ direction. ${ }^{26}$

In Figs. 1(a)-1(d), it is evident that the first and second Fe layer growth is largely mediated by the step-flow growth mechanism. The deposited atoms are in most cases sufficiently mobile that they cross the surface terraces and are incorporated at upward-facing step-edges. This leads to an outward propagation or "flow" of the step over the underlying terrace. To our knowledge this was unobserved in previous STM studies of the $\mathrm{Fe} / \mathrm{Mo}(110)$ and $\mathrm{Fe} / \mathrm{W}(110)$ 

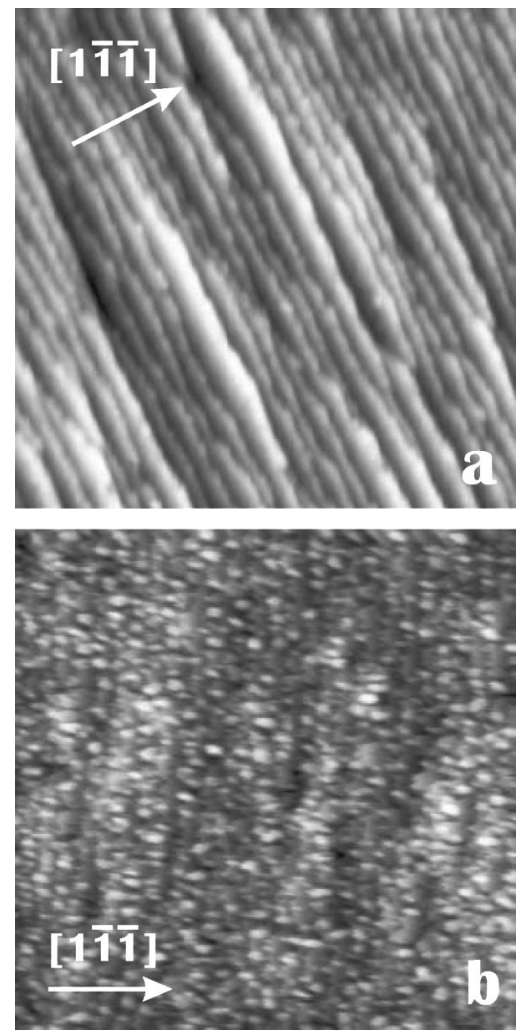

FIG. 2. (a) $600 \times 600 \AA^{2}$ STM image of a vicinal Mo(110) surface; oriented to within $\sim 4.6^{\circ}$ of the surface normal, this yields an average terrace width of the order of $30 \AA$. The step-edge direction runs roughly perpendicular to the [1 $\overline{1} \overline{1}]$ crystallographic direction. (b) $600 \times 600 \AA^{2} \mathrm{STM}$ image of a $0.6 \mathrm{ML}$ Fe film grown on the vicinal surface at $300 \pm 15 \mathrm{~K}$. The substrate terraces are decorated with monolayer thick islands with a mean diameter of 10-20 ̊.

systems in this regime. ${ }^{18,27}$ In these earlier studies, growth occurred through the nucleation of two-dimensional Fe islands on the substrate terraces, with gradual coalescence of these islands into a uniform layer. One likely explanation for this difference is the slightly higher temperature of the substrate $(300 \leqslant T \leqslant 330 \mathrm{~K})$ during the deposition of these films. This would indicate a high morphological sensitivity to the substrate temperature in this regime.

Another possible explanation for this difference may be the narrower average terrace width obtained on the substrate used here $(\sim 200 \AA)$, compared to the substrates used in these previous studies, which had terrace widths in the 100$1000 \AA$ range. ${ }^{18,27}$ With a narrower terrace width, diffusing Fe adatoms would have a higher arrival rate at substrate steps and therefore an increased rate of incorporation at the step edges. The effects of step density and terrace width can be considered by examining the growth of $\mathrm{Fe}$ on a vicinal Mo(110) surface such as that shown in Fig. 2(a). In this case, the substrate was oriented at an angle of $\sim 4.6^{\circ}$ to the surface normal, which yielded terraces with an average terrace width of $\sim 30 \AA$. The terraces in Fig. 2(a) are separated by

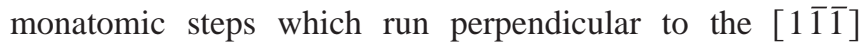
crystallographic direction. This vicinal surface was cleaned in a similar fashion to the low-index surface described above; more details on the preparation and growth of $\mathrm{Fe}$
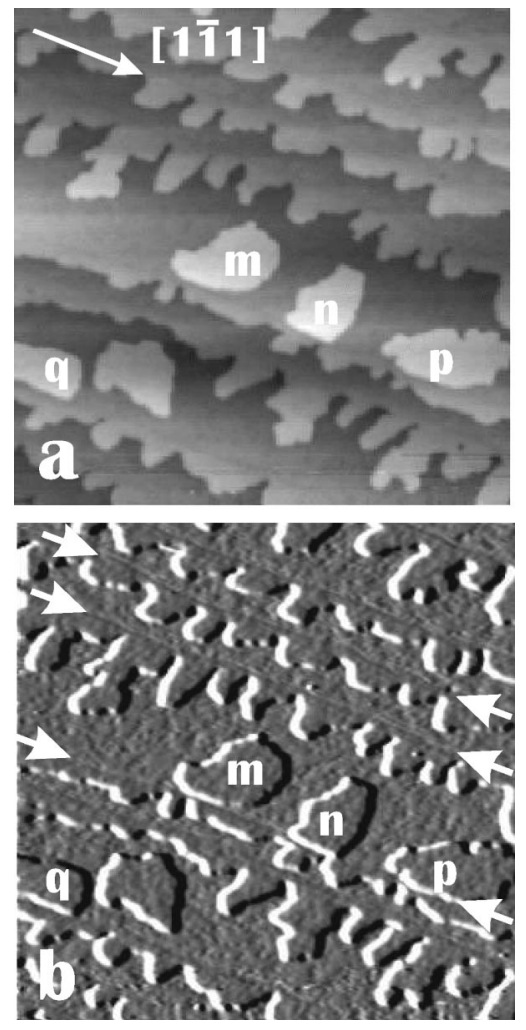

FIG. 3. (a) $1000 \times 1000 \AA^{2}$ topographic STM image of a 0.42 ML Fe film grown at $330 \pm 15 \mathrm{~K}$. (b) The same image has been differentiated to enhance the contrast. The arrows in (b) highlight the positions of dark lines representing fractional steps. These appear where substrate steps have been decorated with Fe by stepflow growth. The monolayer islands $m, n, p$, and $q$ grow on substrate terraces along the inside of the fractional steps, but do not cross them to grow on the Fe decorating the outside. This occurs because of the additional cost in elastic energy associated with maintaining pseudomorphic growth on top of another Fe layer.

films on this surface were given in Refs. 19, 28, and 29. Figure 2(b) shows the results of depositing 0.6 ML Fe on this surface. The substrate terraces are decorated with pseudomorphic monolayer islands with a mean diameter of 10-20 А. There is no evidence of step decoration (i.e., stepflow growth) similar to that displayed in Figs. 1(a)-1(d). It can therefore be concluded that the step decoration observed in Figs. 1(a)-1(d) is not simply due to the narrow width of the substrate terraces. However, the influence of the step orientation must also be considered. The steps in Fig. 1 are oriented parallel to the $[1 \overline{1} 1]$ direction, while those in Fig. 2 are oriented perpendicular to the $[1 \overline{1} \overline{1}]$ direction. The orientation of the steps on the surface has implications for the sticking probability at the step edges, the barriers to interlayer diffusion over the steps and the diffusion of $\mathrm{Fe}$ adatoms along the steps. It has been shown experimentally that the orientation of the substrate steps can have a dramatic effect on the growth of Fe on the W(110) surface. ${ }^{15}$

In the submonolayer coverage range, the decoration of the substrate steps by Fe can be distinguished by the presence of a fractional step of $0.2-0.4 \AA$ height at the Fe-Mo boundary; this is highlighted in Fig. 3. This fractional step is also 
observed in the $1.0 \leqslant \theta \leqslant 1.8$ ML coverage range, where it marks the transition between the first and second Fe layers at a substrate step. The fractional step may be due to a difference in either the local density of states or the atomic radii of both metals.

While growth of the first Fe layer occurs mainly by the step-flow mechanism, two-dimensional $\mathrm{Fe}$ islands are formed on some of the substrate terraces, as shown in Fig. 1(a). The size and number of these islands is generally found to increase with increasing terrace width. Fe atoms deposited on a wider terrace spend longer diffusing about and therefore have a higher probability of nucleation on the terrace. In addition, islands that grow on the wider terraces grow to large sizes as they are not rapidly incorporated into the encroaching step-flow growth. Where these islands reach a substrate step, the overlap of $\mathrm{Fe}$ growth on $\mathrm{Fe}$ appears to be inhibited. Instead, the islands grow parallel to the step (along the [111] direction), as shown in Fig. 3. A similar effect is observed for the growth of second layer $\mathrm{Fe}$ islands [Fig. 1(c)]. This occurs despite the presence of an anisotropic behavior [evident in Figs. 1(e) and 1(f)] that favors island growth across the step, along the $[00 \overline{1}]$ direction. The effect may be explained by considering the balance of surface, interface, and elastic energies involved during pseudomorphic growth. The first Fe layer can wet the Mo(110) surface, despite the cost in elastic energy, because of the lower surface energy of $\mathrm{Fe}\left(\gamma_{\mathrm{Fe}}=2.55 \mathrm{~J} \mathrm{~m}^{-2}\right)$ compared to Mo $\left(\gamma_{M o}=2.95 \mathrm{~J} \mathrm{~m}^{-2}\right) .{ }^{16}$ However, subsequent layers that are deposited, experience a lower binding energy because of the Fe layer already present on the $\mathrm{Mo}(110)$ surface. In addition, there is an increased cumulative cost in elastic energy associated with maintaining pseudomorphic growth. As a result, the overlap of one strained Fe layer on top of another is not favored, though overlap can occur if the elastic strain in the upper Fe layer is relieved. This can occur either by the formation of dislocations, or, if the area of the overlap is small, by the relaxation of atom positions along the edges of the layer.

Because of the comparatively large lattice mismatch between $\mathrm{Fe}$ and Mo (8.9\%), only the first layer of the film maintains pseudomorphic registry with the underlying substrate. Dislocation lines appear along the [001] direction early in the formation of the second layer. They are present in Fig. 1 at a coverage of 1.6 ML. Many of the dislocations are initiated at kink and vacancy sites in the second Fe layer. This suggests that the dislocations are formed in the regions where the film grows together and coalesces to form a closed layer. Previous LEED and STM studies of the Fe/W(110) and $\mathrm{Fe} / \mathrm{Mo}(110)$ systems also found evidence of dislocations propagating along the [001] direction in films in the $1.7 \leqslant \theta$ $\leqslant 1.9$ ML coverage range. ${ }^{18,27,30}$ These dislocations cause the film to relax along the orthogonal [1 $\overline{1} 0]$ direction. The film relaxes along the [1 $1 \overline{0} 0]$ direction first because of the higher cost in energy associated with elastic strain of the film lattice along this direction. ${ }^{26}$

From the STM image in Fig. 4(a), it can be seen that the corrugation of the dislocation is highest $(0.4 \pm 0.1 \AA)$ over a
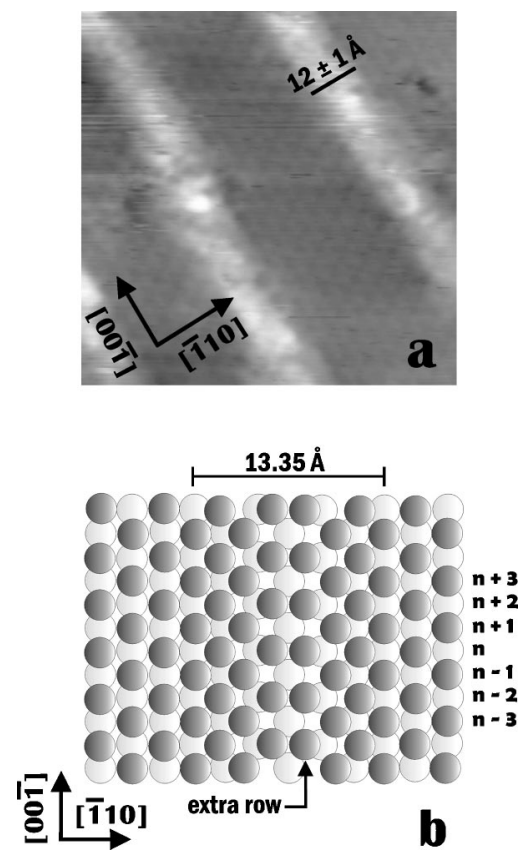

FIG. 4. (a) $90 \times 90 \AA^{2}$ STM image showing dislocation lines in the second layer of a $1.8 \mathrm{ML}$ Fe film deposited $340 \pm 15 \mathrm{~K}$. (b) A model of the dislocation process due to the insertion of an extra row of Fe atoms along the [001] direction. The first layer Fe atoms are light gray, while the second layer Fe atoms are dark gray.

length of $12 \pm 1 \AA$ along the [ $\overline{1} 10]$ direction. This indicates that most of the lattice distortion produced by the dislocation occurs over this scale. Outside this region, the lattice distortion falls off with increasing distance from the dislocation. The atomic resolution visible in Fig. 4(a) is an oxygeninduced $p(2 \times 2)$ reconstruction. A model for the dislocation is proposed in Fig. 4(b), where most of the lattice distortion occurs over a comparable length to the measured value. Because the Mo substrate holds the Fe film in tensile strain, the dislocations are formed by the insertion of an extra row of $\mathrm{Fe}$ atoms into the second $\mathrm{Fe}$ layer along the [001] direction. The model assumes that in the second layer of film growth, the $\mathrm{Fe}$ lattice remains pseudomorphic along the $[00 \overline{1}]$ direction. Because of the centered-rectangular symmetry of the underlying surface, the extra $\mathrm{Fe}$ atoms are only inserted into every second [1 $\overline{1} 0]$ atomic row. This can be seen in Fig. 4(b), where the second layer rows labeled $n$ $-2, n$, and $n+2$ contain one more $\mathrm{Fe}$ atom than rows $n$ $-3, n-1, n+1$, and $n+3$. The extra $\mathrm{Fe}$ atoms in rows $n$ $-2, n$, and $n+2$ cause a relaxation of the interatomic spacing along the [1 $1 \overline{1} 0]$ direction of each row. The displacement of the atoms in these rows induces a relaxation of the atom positions in rows $n-3, n-1, n+1$, and $n+3$, even though these rows do not have extra Fe atoms. The lateral offset of the atoms from the pseudomorphic lattice positions along the [1 $1 \overline{1} 0]$ direction establishes a strain field about each dislocation line. These strain fields contribute to the diffusion and nucleation kinetics of $\mathrm{Fe}$ adatoms deposited in the vicinity of the dislocations, by changing the local periodic potential experienced by the adatoms due to the surface. As a result, the 


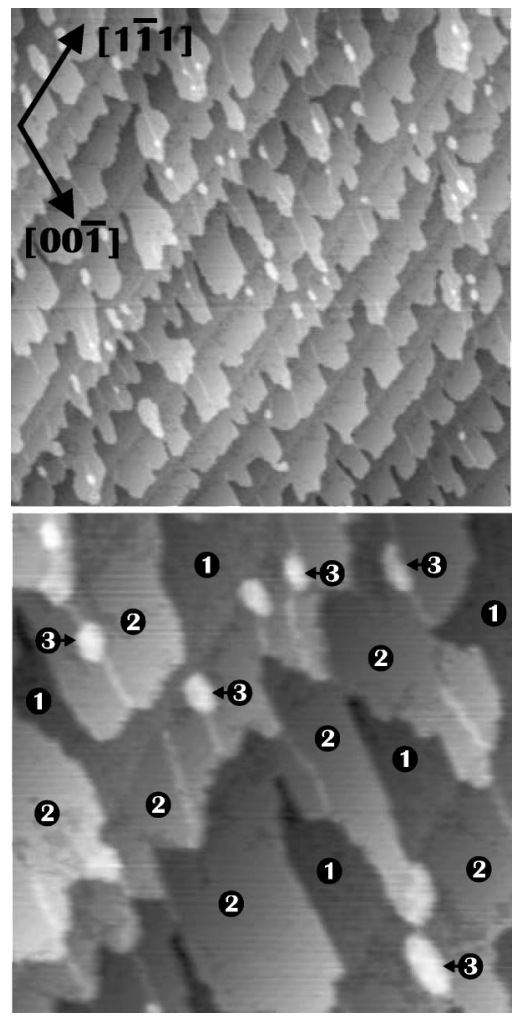

FIG. 5. (a) $2000 \times 2000 \AA^{2}$ STM image of a 1.8-ML film, showing the preferential growth of third layer Fe islands at the positions of dislocations in the second Fe layer. (b) $615 \times 615 \AA^{2}$ zoom-in image; the local Fe layer thickness is indicated. The white lines running along the $[00 \overline{1}]$ direction are dislocation lines in the second Fe layer. It is clear that many of the third layer Fe islands grow on top of these dislocations.

dislocations form favorable nucleation sites for islands of successive Fe layers. This can be seen in Fig. 5, where the formation of third layer Fe islands occurs almost exclusively at the sites of dislocations in the second Fe layer.

In the third Fe layer, the increased elastic energy in the film leads to the abrupt formation of a two-dimensional dislocation network. The onset of this network can be seen in Figs. 6(a) and 6(b), where the local film thickness increases from two to three layers as the Fe film crosses a buried substrate step. The surface corrugation due to the dislocation network is evident on the areas of the film that reach a thickness of three layers. It should be noted that the fractional step observed in the $0 \leqslant \theta \leqslant 1.8 \mathrm{ML}$ coverage range, is absent for the thickness transition between the second and third Fe layers in higher coverage films. The dislocation network becomes widespread during the transition to StranskiKrastanov growth, as shown in Fig. 6(c). LEED patterns of the films in this coverage range display a set of satellite spots like those shown in Fig. 6(d), which are produced by the periodic lattice distortions in the film. This effect has also been observed on films grown under similar conditions in the $\mathrm{Fe} / \mathrm{W}(110)$ system. ${ }^{30}$

An example of this dislocation network is resolved in the STM image shown in Figs. 7(a) and 7(b). The abrupt onset of the two-dimensional dislocation with the thickness
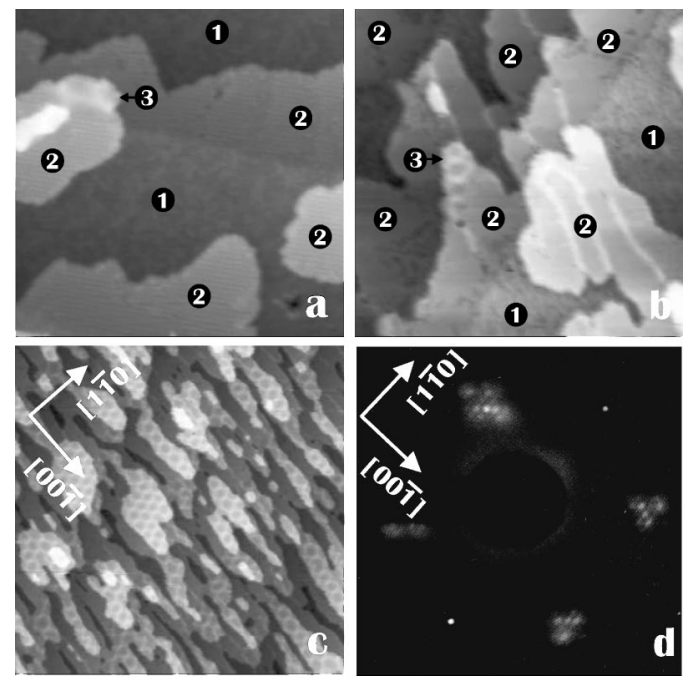

FIG. 6. STM images showing the onset of a two-dimensional dislocation network, where Fe layers overlap to form a local thickness of three layers or more. (a) $400 \times 400 \AA^{2}$ on a $1.6 \mathrm{ML}$ film grown at $300 \pm 15 \mathrm{~K}$ and (b) $1000 \times 1000 \AA^{2}$ on a $1.8 \mathrm{ML}$ film grown at $340 \pm 15 \mathrm{~K}$. Where the local film thickness reaches three layers, the surface corrugation due to the two-dimensional dislocation network appears. (c) $1000 \times 1000 \AA^{2}$ on a $2.4 \mathrm{ML}$ film grown at $325 \pm 15 \mathrm{~K}$. The dislocation network becomes well-established as the film adopts the Stranski-Krastanov growth mode at higher coverages. (d) LEED pattern with satellite spots produced by the dislocation network on a $4.7 \mathrm{ML}$ film grown at $320 \pm 15 \mathrm{~K}, E_{\text {beam }}$ $=123 \mathrm{eV}$.

transition between the second and third layers of the film is evident in these images. Figure 7(c) shows a zoom in of a three layer thick Fe island; a derivative image is used to
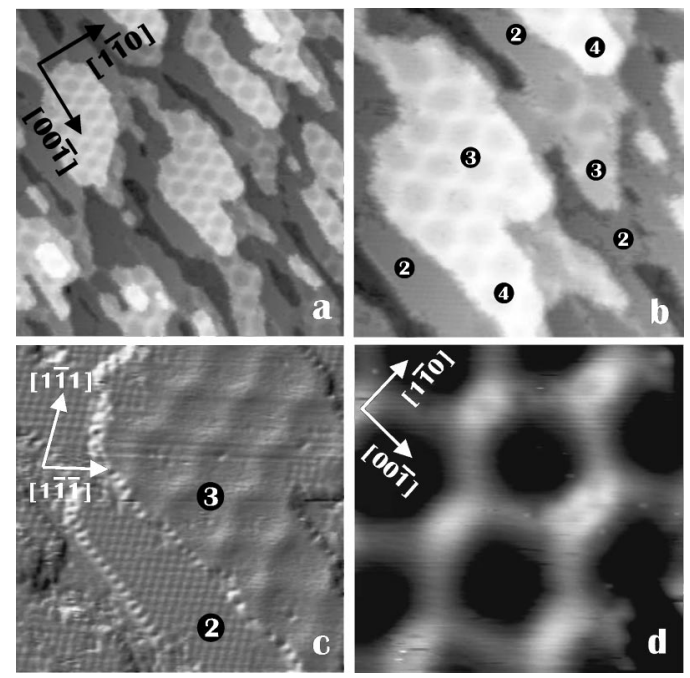

FIG. 7. (a) $600 \times 600 \AA^{2}$ STM image of a 2.4 ML film grown at $325 \pm 15 \mathrm{~K}$. (b) $300 \times 300 \AA^{2}$ zoom-in with the local Fe layer thickness indicated. (c) $200 \times 200 \AA^{2}$ derivative image showing the atomic structure of an oxygen-induced $p(2 \times 2)$ reconstruction on the second $\mathrm{Fe}$ layer and the relative orientation of the twodimensional dislocation network on the third Fe layer. (d) 100 $\times 100 \AA^{2}$ contrast-enhanced image showing the detailed structure of the dislocation network on the third Fe layer in image (c). 


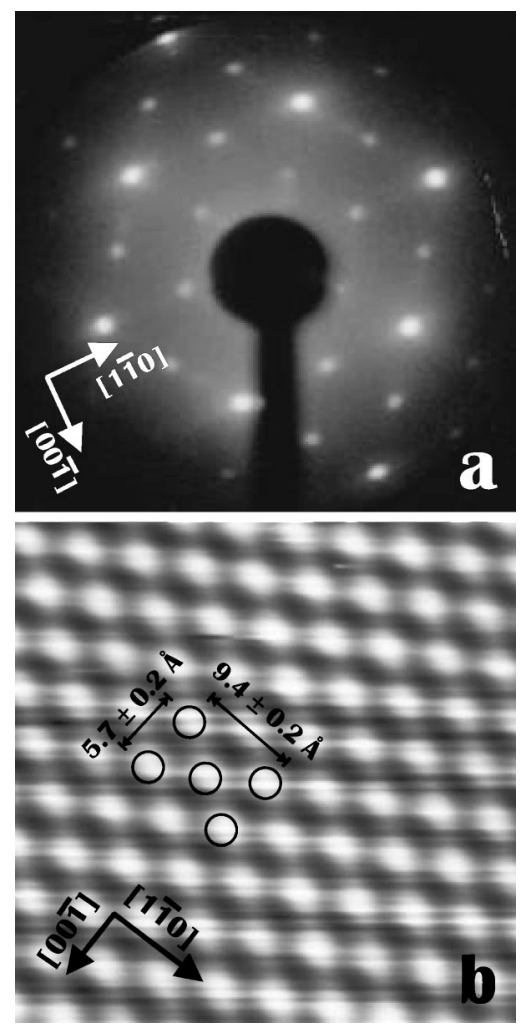

FIG. 8. (a) LEED diffraction pattern of a 1.2 ML Fe film grown around $300 \mathrm{~K}$; taken with a primary beam energy of $123 \mathrm{eV}$. The pattern is identified as a $p(2 \times 2)$ with respect to the primitive unit cell of the bcc (110) surface. The integral-order spots appear the brightest, while fainter half-order spots appear between them. (b) $50 \times 50 \AA^{2}$ STM image of the surface of a second layer Fe island, showing the structure of the oxygen-induced $p(2 \times 2)$ reconstruction. The non primitive (centered-rectangular) unit cell of the reconstruction is outlined; the dimensions of this cell were measured to be $5.7 \pm 0.2 \AA$ and $9.4 \pm 0.2 \AA$ along the $[00 \overline{1}]$ and $[1 \overline{1} 0]$ directions, respectively.

highlight the structure of the dislocation network. A $p(2 \times 2)$ reconstruction [identified with respect to the primitive unit cell of the bcc (110) surface] is atomically resolved on top of the second Fe layer surrounding the island. The STM image in Fig. 8(b) shows the structure of this reconstruction in greater detail; the structure is resolved on top of a second layer Fe island on a 1.6 ML film grown around 300 $\mathrm{K}$. This structure repeats the centered-rectangular symmetry of the underlying lattice with a periodicity of $5.7 \pm 0.2 \AA$ along the $[00 \overline{1}]$ direction and $9.4 \pm 0.2 \AA$ along the $[1 \overline{1} 0]$ direction. The packing density of this structure is highest along the $[1 \overline{1} 1]$ and $[1 \overline{1} \overline{1}]$ directions, which are marked in Fig. 7(c) for reference. An AES analysis indicated that after an Fe film has been deposited, oxygen is adsorbed on the film from the residual gas in the UHV system. In the 0.4 $\leqslant \theta \leqslant 1.8 \mathrm{ML}$ coverage range, this results in a transition in the LEED pattern of the film from a $p(1 \times 1)$ to a $p(2 \times 2)$ pattern, such as that shown in Fig. 8(a). A similar structure was previously reported on the $\mathrm{Fe}(110)$ surface at a $0.25 \mathrm{ML}$ coverage of oxygen, where it was identified as a $c(2 \times 2)$ reconstruction formed with respect to the non-primitive unit cell of the bcc (110) surface. ${ }^{31,32}$
This structure provides a useful reference by which the orientation and scale of the dislocation network in the third and fourth Fe layers may be visualized. The structure on top of the Fe island is resolved in greater detail in Fig. 7(d); the image is contrast enhanced to highlight the arrangement of the dislocations in the two-dimensional network. The network is comprised of an array of regions of enhanced corrugation that reproduce the centered-rectangular symmetry of the underlying $\operatorname{Mo}(110)$ surface. These regions of enhanced corrugation are formed by the overlap of a network of dislocation lines that run along the [1 $\overline{1} 1]$ and [1 $\overline{1} \overline{1}]$ directions, which can be seen when comparing Figs. 7(c) and 7(d). The regions where the dislocations overlap have a corrugation amplitude of $0.4-0.8 \AA$ and have full-width at half-maximum dimensions of $12 \pm 1 \AA$ along the [001] direction and $20 \pm 1 \AA$ along the [1 $1 \overline{1} 0]$ direction.

Mean values for the periodicity of the network along the $[00 \overline{1}]$ and $[1 \overline{1} 0]$ directions in the third Fe layer were determined from STM images of this film. These were found to be $d_{[00 \overline{1}]}=35 \AA$ and $d_{[1 \overline{1} 0]}=57 \AA$, respectively. These values correspond to the matching of $12 \mathrm{Fe}$ atoms for every $11 \mathrm{Mo}$ atoms along the [001] direction, and $14 \mathrm{Fe}$ atoms for every 13 Mo atoms along the [1]̄0] direction. The irregular shape of many of the Fe islands distorts the symmetry and periodicity of the dislocation network. For example, many of the $\mathrm{Fe}$ islands are elongated along the [001] direction because of anisotropic growth. When the periodicity of the dislocation network on these islands is measured, it is found that the periodicity along the $[00 \overline{1}]$ direction is typically larger than the mean value measured. The dislocation density within these elongated islands is lower than the mean value because there is another mechanism working to relieve the strain. Part of the elastic strain within the islands is relieved by the relaxation of the positions held by Fe atoms along the edges of the islands - the influence of this mechanism is most felt near the island edges. Far away from the island edges this mechanism has little influence and the strain can only be relieved by dislocation formation. As a result, where the islands are large and have a more isotropic shape, the periodicity of the network is closer to the mean values measured.

\section{B. Elevated-temperature growth}

At elevated temperatures $(495 \leqslant T \leqslant 525 \mathrm{~K})$, the first and second Fe layers are formed by the step-flow mechanism, resulting in the formation of uniformly wide Fe stripes along the substrate steps ([1 11$]$ direction). STM images of Fe stripes at 1.2, and 1.5-ML coverages are shown in Figs. 9(a) and 9 (b) respectively. In both cases, the substrate terraces are completely covered by a closed pseudomorphic monolayer, while the remaining $\mathrm{Fe}$ decorates the terrace steps. The second layer Fe can be distinguished in the STM images by the presence of fractional steps that run along the [1 11$]$ direction; these steps are marked with arrows in Fig. 9. The first Fe layer in both images is defect free, but randomly spaced dislocations (which appear as white lines in the STM 

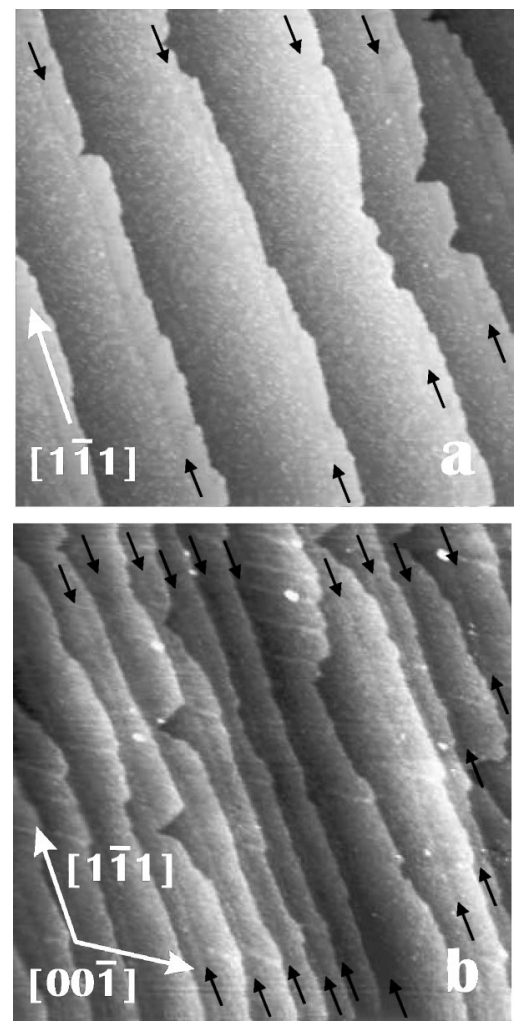

FIG. 9. Iron stripes formed by step-flow growth on $\mathrm{Mo}(110)$ at elevated temperatures. (a) $1000 \times 1000 \AA^{2}$ STM image of a $1.2 \mathrm{ML}$ film grown at $495 \pm 15 \mathrm{~K}$. (b) $2000 \times 2000 \AA^{2}$ image of a $1.5 \mathrm{ML}$ film grown at $525 \pm 15 \mathrm{~K}$. The first Fe layer is completely closed and free of dislocations. The fractional steps marking the transition between the first and second Fe layers are marked by arrows. Randomly spaced dislocations are formed in some of the wider second layer stripes in image (b); these appear as white lines that run along the $[00 \overline{1}]$ direction.

image) are formed along the [001] direction in the second layer in Fig. 9(b), where the average stripe width is 130-200 $\AA$. These dislocations are not present in the second layer in Fig. 9(a), where the average stripe width is $30-60 \AA$. It is clear that dislocations only appear in the second layer stripes above a critical stripe width of the order of $100 \AA$. Below this width, the relaxation of the atom positions at the outer edge of each stripe relieves a sufficient amount of the pseudomorphic strain within the stripe, so that dislocations are prevented from forming.

There is a significant transformation in the film morphology at higher coverages, where the films are characterized by large, well-separated Fe islands, which are supported on a closed pseudomorphic Fe layer (Fig. 10). The presence of this closed monolayer was detected by an analysis of the area and local thickness of islands in STM images of the films, and a comparison with the amounts deposited for each film as measured by the quartz crystal balance. The presence of a pseudomorphic Fe monolayer was also observed on similar films grown on W(110). ${ }^{33,34}$ The Fe islands on top of this layer have a well-defined shape, where their edges are generally straight and lie along a low-index crystallographic direction such as the [1 $\overline{1} 1]$ and [00 $\overline{1}]$ direc-
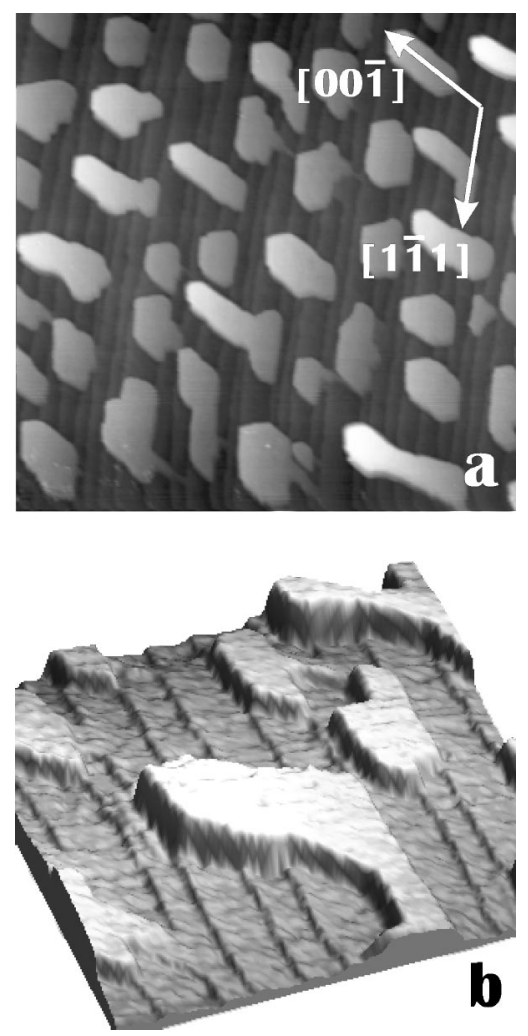

FIG. 10. (a) $5000 \times 5000 \AA^{2}$ image of a $2.4 \mathrm{ML}$ film grown on $\mathrm{Mo}(110)$ at $515 \pm 15 \mathrm{~K}$. (b) A three-dimensional representation of the Fe islands, showing the wedge shape formed as they propagate over several terraces while maintaining a flat (110) surface.

tions. The islands are slightly elongated along the [001] direction, indicating a growth anisotropy that favors this direction. While propagating across several terraces, the surface of each island is an unbroken (110) atomic plane, so that the local thickness of the island increases by one layer for each substrate step crossed. As a result, the islands have a distinctive wedge-shaped cross section (Fig. 10). This effect was observed in STM studies of films grown under similar conditions in both the $\mathrm{Fe} / \mathrm{W}(110)$ and $\mathrm{Fe} / \mathrm{Mo}(110)$ epitaxial systems, and was attributed to surface free energy minimization. ${ }^{18,19,27}$

Because the local thickness of these wedge-shaped islands increases continuously, they present an excellent opportunity to study the strain relaxation processes occurring within the film. A STM study of Fe/W(110) growth $^{27}$ found that an array of parallel dislocation lines, with a typical separation of $46 \AA$, were formed along the [001] direction in the second layer of each island. In the third Fe layer the separation of the lines decreased to $27 \AA$, while a two-dimensional dislocation network was formed in the fourth Fe layer. A previous STM study of Fe/Mo(110) growth, ${ }^{18}$ indicated that dislocation lines were formed in the second Fe layer of each island, while a two-dimensional dislocation network was formed in the third monolayer. The results presented here are in closer agreement with the observations for Fe/W(110) growth, ${ }^{27}$ rather than what has been previously observed for $\mathrm{Fe} / \mathrm{Mo}(110)$ growth. ${ }^{18}$ There is no evidence of dislocation formation in the first $\mathrm{Fe}$ layer, while randomly spaced 


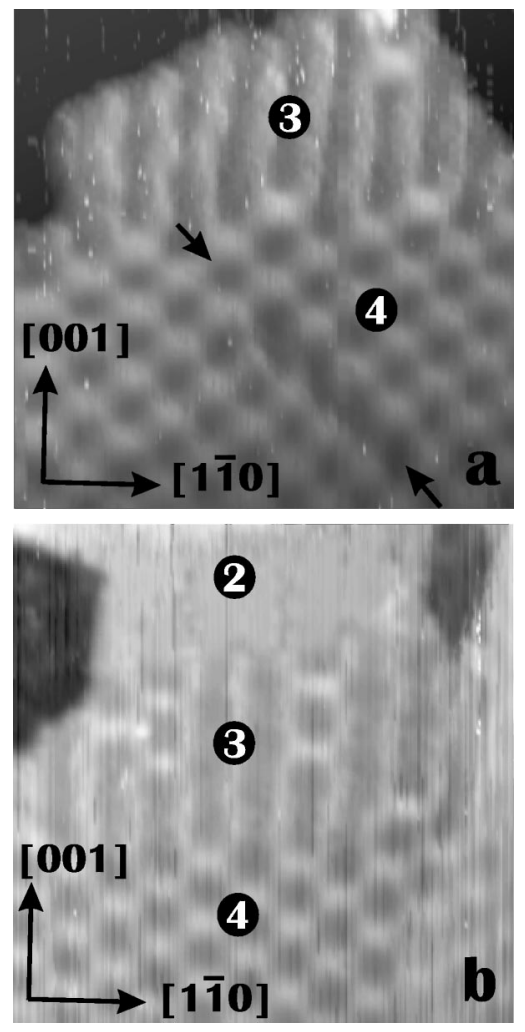

FIG. 11. (a) and (b) $300 \times 300 \AA^{2}$ STM images showing the onset of dislocation networks from an array of closely spaced dislocation lines as the local thickness of the Fe island increases from 2 to 3 ML. Mismatch defects may be formed in the dislocation network where adjacent network domains grow into one another. One such defect is indicated by the arrows in image (a).

dislocations are formed in the second Fe layer. An array of closely-spaced dislocation lines is observed in the third Fe layer of each island. The typical separation between the lines along the [1 $1 \overline{1} 0]$ direction is $30 \pm 2 \AA$. The corrugation and full-width at half-maximum of these lines is comparable to the that of the lines shown in Fig. 4. STM images obtained on the flat (110) surface of these islands show how a twodimensional dislocation network is formed by the overlap between these dislocation lines as the local island thickness increases from three to four monolayers thick (Fig. 11). In some cases, the early onset of dislocations along the [1 $1 \overline{1} 0]$ direction is observed in the third Fe layer. This occurs when adjacent dislocation lines get too close to one another i.e. when they have a separation of less than $30 \AA$. In this case, the strain fields associated with each dislocation line overlap, prompting the formation of a dislocation line along the [1 $1 \overline{1} 0]$ direction. As a result, it can be concluded that while the majority of the lattice distortion produced by each dislocation is localized over $\sim 12 \AA$, the total strain field produced by the dislocation extends by roughly $15 \AA$ to either side, along the [1 $1 \overline{0} 0]$ direction.

Mean values for the periodicity of the network were found to be $33 \AA$ along the [00 $\overline{1}]$ direction and $54 \AA$ along the $[1 \overline{1} 0]$ direction. These values correspond to the matching of $10.5 \mathrm{Mo}$ atoms to $11.5 \mathrm{Fe}$ atoms along the [001] direction and $12 \mathrm{Mo}$ atoms to $13 \mathrm{Fe}$ atoms along the [1 $\overline{1} 0]$ direction. Therefore, while this network is similar to the dislocation network observed on films grown at $300 \mathrm{~K}$, it shows a greater degree of relaxation along both the major crystallographic directions. In Fig. 11(a), a mismatch boundary can be observed between adjacent domains of the dislocation network. This demonstrates that the dislocation network may start from a number of separate points in the fourth $\mathrm{Fe}$ layer and that the domains subsequently grow into one another in the thicker parts of the island.

The dislocation network is first established in the fourth Fe layer of each island. The corrugation amplitude of the network at this island thickness was measured to be 0.5 $\pm 0.1 \AA$ along both the $[00 \overline{1}]$ and $[1 \overline{1} 0]$ directions. Toward the thicker end of each island ( $\sim 10 \mathrm{ML})$, the corrugation amplitude of the dislocation network decreases slightly. The effect is more pronounced on Fe islands grown on vicinal Mo(110), where the island thickness range can be much greater. ${ }^{19}$ With each additional layer of the island, more dislocations are added, the periodicity of the dislocation network decreases and the atom packing density of each layer increases until the film lattice approaches that of unstrained Fe. The largest increase in the number of dislocations occurs in the second to fourth layers of the islands. For each subsequent layer, only a few additional dislocations are introduced into the dislocation network, so that the relaxation of the film lattice to unstrained $\mathrm{Fe}$ occurs gradually over many Fe layers. This behavior is reflected in a study of the mechanical stress of Fe films deposited on W(110), ${ }^{34}$ where the film stress was observed to decrease drastically upon the formation of dislocations, but beyond the initial point of dislocation formation, the decrease in film stress was observed to be very gradual. Because the dislocation density per Fe layer does not increase significantly beyond the fourth layer, the periodicity of the dislocation network does not appear to decrease significantly from layer to layer.

Figure 12 compares LEED patterns of two Fe films grown at elevated temperatures on Mo(110). Figure 12(a) shows the LEED pattern produced by the islands shown in Fig. 10; a $2.4 \mathrm{ML} \mathrm{Fe}$ film grown at $515 \pm 15 \mathrm{~K}$. The islands are not very thick (6-10 ML maximum local thickness) and the upper layers contain some residual strain. The film displays a set of satellite spots due to the presence of the twodimensional dislocation network in the upper layers of the islands (not resolved in the STM images). It should be noted that the satellite spots in this pattern are much sharper than those obtained on films grown near room temperature [cf. Fig. 6(d)], indicating that the dislocation network is more fully developed in films grown at elevated temperatures. A similar pattern has been reported by He et al. $^{35}$ for a $1.3 \mathrm{ML}$ Fe film deposited on $\mathrm{Mo}(110)$ at $115 \mathrm{~K}$ and subsequently annealed to $540 \mathrm{~K}$. Faint half-order spots are visible halfway between the integral-order spots in Fig. 12(a); these may be due to the presence of a $2 \times 2$ reconstruction on the closed pseudomorphic monolayer that covers the substrate between the islands. It is possible that this is the same $p(2 \times 2)$ reconstruction as that observed on films grown around 300 K. Figure 12(b) shows the LEED pattern observed on a 


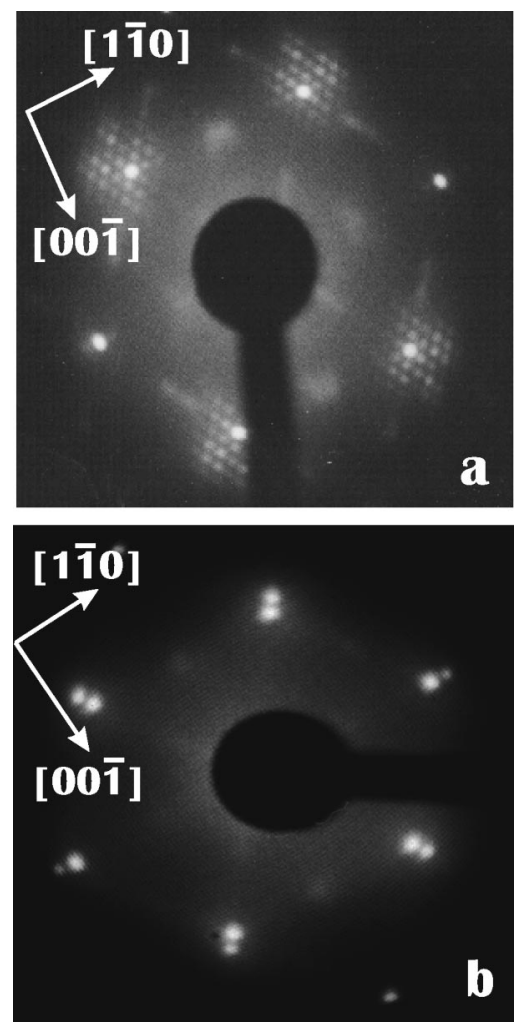

FIG. 12. (a) $12 \times 12$ LEED pattern produced by the twodimensional dislocation network in a $2.4 \mathrm{ML}$ Fe film grown on $\mathrm{Mo}(110)$ at $515 \pm 15 \mathrm{~K}$. $E_{\text {beam }}=111 \mathrm{eV}$. (b) The LEED pattern produced by a $3.5 \mathrm{ML}$ Fe film grown at $700 \pm 15 \mathrm{~K}$. The first order spots of the $\mathrm{Fe}(110)$ film surface lie outside those of the pseudomorphic monolayer covering the $\mathrm{Mo}(110)$ substrate. $E_{\text {beam }}$ $=94 \mathrm{eV}$.

3.5 ML film grown at $700 \pm 15 \mathrm{~K}$. Because of the deposition temperature, the islands in this film are much larger and thicker (15-20 ML) and the upper layers of the islands are unstrained. The LEED pattern in Fig. 12(b) is consistent with the unstrained $1 \times 1 \mathrm{Fe}(110)$ surface of the islands superimposed on that of the pseudomorphic monolayer covering the substrate between the islands.

\section{Spin-polarized STM}

It has been shown that Fe films in the $0 \leqslant \theta \leqslant 2$ ML coverage range, grown on $\mathrm{Mo}(110)$ at elevated temperatures, produce step-oriented arrays of 1-2 ML thick Fe stripes. Morphologically, these $\mathrm{Fe} / \mathrm{Mo}(110)$ films are very similar to those observed for Fe films in the same coverage range and grown under similar conditions on W(110). Detailed studies of the magnetic properties of these Fe/W(110) films have shown that dipolar superferromagnetic and antiferromagnetic coupling can occur between the Fe stripes, depending on the stripe arrangement. ${ }^{10,36}$ The latter phenomenon has recently been used to demonstrate spin-polarized scanning tunneling spectroscopy (SPSTS). ${ }^{11,12}$ It is reasonable to expect that Fe stripes grown on $\mathrm{Mo}(110)$ will be a good candidate for similar spin-polarized STM/STS experiments.

The wedge-shaped island structures observed in Fe films grown at elevated temperatures on both $\operatorname{Mo}(110)$ and
$\mathrm{W}(110)$, may also be an interesting system for spin-polarized STM studies. The magnetic properties of this structure should change in a predictable way on the nanometer scale. It has been observed that while the first Fe layer on W(110) is not ferromagnetic at room temperature, the second $\mathrm{Fe}$ layer is. ${ }^{7,8}$ The wedge-shaped Fe islands provide a gradually increasing local film thickness, while maintaining a flat $\mathrm{Fe}(110)$ surface that is unbroken by steps. By scanning this surface at room temperature with a ferromagnetic or antiferromagnetic STM tip ${ }^{37-40}$ it may be possible to obtain topographic contrast at the thin end of each $\mathrm{Fe}$ island on the basis of the different magnetic order of the first and second layers. This contrast would not be apparent in STM images taken at temperatures below the Curie temperature of the first Fe layer.

\section{CONCLUSIONS}

The growth mechanism of low coverage Fe films on Mo(110) depends sensitively on the substrate temperature. Fe first and second layer growth in the $300 \leqslant T \leqslant 345$ temperature range was found to adopt a step-flow mechanism rather than the two-dimensional island growth observed in previous studies of $\mathrm{Fe} / \mathrm{Mo}(110)$ and $\mathrm{Fe} / \mathrm{W}(110)$ epitaxy. ${ }^{18,27}$ The large lattice mismatch $(8.9 \%)$ means that only the first Fe layer is pseudomorphic. Dislocation lines are formed along the $[00 \overline{1}]$ direction in the second layer. These dislocations act as favorable sites for nucleation of third layer islands. The one-dimensional dislocation lines are formed by the insertion of an extra row of Fe atoms along the $[00 \overline{1}]$ direction. There is a transition from layer-by-layer to Stranski-Krastanov growth at coverages above 1.8 ML, with increasing anisotropy of growth observed along the [11̄0] crystallographic direction. A dislocation network is formed in the third Fe layer due to the overlap of the dislocation lines formed along the $\langle 1 \overline{1} 1\rangle$ set of directions. For elevated substrate temperatures $(495 \leqslant T \leqslant 700 \mathrm{~K})$, the first and second film layers grow by the step-flow mechanism, forming arrays of parallel, step-oriented stripes. The first layer is pseudomorphic, but randomly spaced dislocations are formed along the $[00 \overline{1}]$ direction in the second layer, when the stripe width exceeds $\sim 100 \AA$. At higher film coverages, large wedge-shaped $\mathrm{Fe}$ islands are formed, which are supported on a closed Fe monolayer. An array of closelyspaced dislocation lines is formed in the third $\mathrm{Fe}$ layer of these islands. This array develops into a two-dimensional dislocation network when the local thickness of the island reaches $4 \mathrm{ML}$. A comparison of the $\mathrm{Fe} / \mathrm{Mo}(110)$ and $\mathrm{Fe} /$ $\mathrm{W}(110)$ systems shows that the strain-relief mechanisms and manner of growth adopted in both systems are very similar, leading one to expect that similar magnetic phenomena may occur in both systems. It is proposed that the Fe stripes and wedge-shaped islands produced in $\mathrm{Fe}$ films grown on $\mathrm{Mo}(110)$ at elevated temperatures are candidate systems for spin-polarized STM and STS experiments.

\section{ACKNOWLEDGMENTS}

This work was supported by the Science Foundation of Ireland and the 5th Framework Program of the European Commission under project Magnetude G5RD-CT-199900005 . 
*Corresponding author. Fax: + 3531671 1759. Email address: shmurphy@tcd.ie

${ }^{1}$ U. Gradmann, Handbook of Magnetric Materials, (Elsevier, Amsterdam, 1993), Vol. 7, p. 1 .

${ }^{2}$ F. Himpsel, J. Ortega, G. Mankey, and R. Willis, Adv. Phys. 47, 511 (1998).

${ }^{3}$ J. Larsen and I. Chorkendorff, Surf. Sci. Rep. 35, 163 (1999).

${ }^{4}$ B. Schulz and K. Baberschke, Phys. Rev. B 50, 13467 (1994).

${ }^{5}$ W. O’Brien, T. Droubay, and B.P. Tonner, Phys. Rev. B 54, 9297 (1996).

${ }^{6}$ R. Vollmer, T. Gutjahr-Löser, J. Kirschner, S. van Dijken, and B. Poelsema, Phys. Rev. B 60, 6277 (1999).

${ }^{7}$ N. Weber, K. Wagner, H. Elmers, J. Hauschild, and U. Gradmann, Phys. Rev. B 55, 14121 (1997).

${ }^{8}$ H. Elmers, J. Hauschild, H. Höche, U. Gradmann, H. Bethge, D. Heuer, and U. Köhler, Phys. Rev. Lett. 73, 898 (1994).

${ }^{9}$ H. Fritzsche, H. Elmers, and U. Gradmann, J. Magn. Magn. Mater. 135, 343 (1994).

${ }^{10}$ J. Hauschild, U. Gradmann, and H. Elmers, Appl. Phys. Lett. 72, 3211 (1998).

${ }^{11}$ M. Bode, O. Pietzsch, A. Kubetzka, and R. Wiesendanger, J. Electron Spectrosc. Relat. Phenom. 114, 1055 (2001).

${ }^{12}$ A. Kubetzka, O. Pietzsch, M. Bode, and R. Wiesendanger, Phys. Rev. B 63, 140407 (2001).

${ }^{13}$ T. Dürkop, H. Elmers, and U. Gradmann, J. Magn. Magn. Mater. 172, L1 (1997).

${ }^{14}$ H. Elmers, J. Hauschild, and U. Gradmann, J. Magn. Magn. Mater. 198-199, 222 (1999).

${ }^{15}$ H. Elmers, J. Hauschild, and U. Gradmann, J. Magn. Magn. Mater. 221, 219 (2000).

${ }^{16}$ L. Mezey and J. Giber, Surf. Sci. 117, 220 (1982).

${ }^{17}$ M. Tikhov and E. Bauer, Surf. Sci. 232, 73 (1990).

${ }^{18}$ J. Malzbender, M. Przybylski, J. Giergiel, and J. Kirschner, Surf. Sci. 414, 187 (1998).

${ }^{19}$ J. Osing and I. Shvets, Surf. Sci. 433-435, 440 (1999).

${ }^{20}$ G. Mariotto, M. D'Angelo, J. Kresnin, and I. Shvets, Appl. Surf. Sci. 144-145, 530 (1999).
${ }^{21}$ G. Mariotto, M. D’Angelo, and I. Shvets, Rev. Sci. Instrum. 70, 3651 (1999).

${ }^{22}$ M. Albrecht, H. Fritzsche, and U. Gradmann, Surf. Sci. 294, 1 (1993).

${ }^{23}$ T. Nawrath, H. Fritzsche, and H. Maletta, Surf. Sci. 414, 209 (1998).

${ }^{24}$ U. Köhler, C. Jensen, C. Wolf, A. Schindler, L. Brendel, and D. Wolf, Surf. Sci. 454-456, 676 (2000).

${ }^{25}$ D. Reuter, G. Gerth, and J. Kirschner, Phys. Rev. B 57, 2520 (1998).

${ }^{26}$ B. Clemens, R. Osgood, A. Payne, B. Lairson, S. Brennan, R. White, and W. Nix, J. Magn. Magn. Mater. 121, 37 (1993).

${ }^{27}$ H. Bethge, D. Heuer, C. Jensen, K. Reshöft, and U. Köhler, Surf. Sci. 331-333, 878 (1995).

${ }^{28}$ J. Osing and I. Shvets, J. Magn. Magn. Mater. 198-199, 734 (1999).

${ }^{29}$ J. Osing, S. Murphy, and I. Shvets, Surf. Sci. 454-456, 280 (2000).

${ }^{30}$ U. Gradmann and D. Waller, Surf. Sci. 116, 539 (1982).

${ }^{31}$ A. Wight, N. Condon, F. Leibsle, G. Worthy, and A. Hodgson, Surf. Sci. 331-333, 133 (1995).

${ }^{32}$ H.-J. Kim and E. Vescovo, Phys. Rev. B 58, 14047 (1998).

${ }^{33}$ D. Sander, A. Enders, C. Schmidthals, D. Reuter, and J. Kirschner, J. Magn. Magn. Mater. 177-181, 1299 (1998).

${ }^{34}$ D. Sander, A. Enders, C. Schmidthals, D. Reuter, and J. Kirschner, Surf. Sci. 402-404, 351 (1998).

${ }^{35}$ J.W. He, W. Shea, X. Jiang, and D. Goodman, J. Vac. Sci. Technol. A 8, 2435 (1990).

${ }^{36}$ J. Hauschild, H. Elmers, and U. Gradmann, Phys. Rev. B 57, R677 (1998).

${ }^{37}$ A. Minakov and I. Shvets, Surf. Sci. 236, L377 (1990).

${ }^{38}$ R. Wiesendanger, I. Shvets, D. Bürgler, G. Tarrach, H. Güntherodt, J. Coey, and S. Gräser, Science 255, 583 (1992).

${ }^{39}$ I. Shvets, R. Wiesendanger, D. Bürgler, G. Tarrach, H. Güntherodt, and J. Coey, J. Appl. Phys. 71, 5489 (1992).

${ }^{40}$ S. Murphy, J. Osing, and I. Shvets, Appl. Surf. Sci. 144-145, 497 (1999). 